\title{
Performance of aerogel as Cherenkov radiator
}

\author{
T. Bellunato ${ }^{1}$, M. Calvi, C. Matteuzzi, M. Musy, P. Negri \\ Università di Milano-Bicocca and INFN, Piazza della Scienza 3, 20126 Milano,Italy
}

A. Braem, E. Chesi, C. Hansen, D. Liko, C. Joram, N. Neufeld, J. Séguinot, P. Weilhammer

CERN, Switzerland

A.R. Buzykaev, E.A. Kravchenko, A.P. Onuchin

Budker Institute of Nuclear Physics, Novosibirsk, Russia

A.F. Danilyuk

Boreskov Institute of Catalysis, Novosibirsk, Russia

S. Easo

Rutherford-Appleton Laboratory, Chilton, Didcot OX11 0QX, UK

\section{S. Wotton}

Cavendish Laboratory, University of Cambridge, Madingley Road, Cambridge CB3 0HE, UK

$$
\text { S. Jolly }
$$

Imperial College, London, UK

Keywords: aerogel,Cherenkov,radiator,Hybrid Photon Detectors,angular resolution

\begin{abstract}
Aerogel with index of refraction around 1.03 has been studied as Cherenkov radiator in an experiment at the CERN PS using a $\pi^{-}$and a mixed $\pi^{+} / p$ beam of momenta between 6 and $10 \mathrm{GeV} / \mathrm{c}$. The Cherenkov photons were detected by means of four large HPD tubes designed and constructed at CERN. Results on the photoelectron yield, the Cherenkov angle and its resolution, and the $\pi / p$ separation are obtained. The performances measured demonstrate that a RICH with aerogel is a viable detector for experiments with high multiplicity of particles in the final state.
\end{abstract}

\footnotetext{
${ }^{1}$ also at CERN
} 


\section{Submitted to NIM A}




\section{Introduction}

The usefulness of aerogel as a Cherenkov radiator for RICH detectors was demonstrated a few years ago [1]. Since then, even better quality of aerogel has been produced $[2,3]$. One experiment has built RICH detectors with aerogel (HERMES [4]), and others plan to build one, like LHCb [5] and AMS [6]. The existing detectors (like HERMES [4]) however, needs a Cherenkov angular resolution of the order of 15-20 mrad, while applications to experiments with high multiplicity in the final state need almost an order of magnitude better resolution.

In this note, results are presented from a test made with the CERN-PS beam with different qualities of aerogel, with index of refraction $\mathrm{n} \simeq 1.03$, in view of its application in the LHCb experiment. Results from a previous test [7] have been confirmed and improved. Particle identification is a fundamental requirement for the LHCb experiment. It is essential to distinguish among pions, kaons and protons in a variety of final states of B decays. Meaningful CP violation measurements will be possible only if hadron identification is available to reconstruct final states, and to identify kaons from the b hadron decay to provide an efficient flavour tag.

In the momentum region of $2-10 \mathrm{GeV}$, an aerogel radiator of index of refraction $n=1.03$ can be a suitable solution.

\section{The Aerogel}

Aerogel is a low density material, essentially made of $\mathrm{SiO}_{2}$.

An important optical requirement of a Cherenkov radiator is that it should not scatter the produced photons. Any angular dispersion caused by the radiator medium will reduce the precision on the Cherenkov emission angle. The dominant cause of aerogel image degradation is Rayleigh scattering [8]. The absorption has very little importance.

The light transmission $\mathrm{T}$ at wavelength $\lambda$ through a sample of thickness $\mathrm{L}$ is well described by the expression

$$
T=A \cdot e^{-C L / \lambda^{4}}
$$

where $\mathrm{C}$ is the clarity coefficient and $\mathrm{A}$ is the transmission in the high- $\lambda$ region.

In the test presented in this paper, 2 tiles of $7 \times 8 \times 4 \mathrm{~cm}^{3}$ and $10 \times 10 \times 4 \mathrm{~cm}^{3}$, produced by the Boreskov Institute of Catalysis in collaboration with the Budker Institute of Nuclear Physics in Novosibirsk, were used. They were tested separately and together to form a total thickness of $8 \mathrm{~cm}$.

The optical properties of these aerogel samples have been tested by measuring the light transmission as a function of the wavelength and aerogel thickness. The tiles produced in Novosibirsk are hygroscopic with refractive index (at $\lambda=400 \mathrm{~nm}) 1.0306$ and 1.0298 respectively. From the curves of Fig. 1 the clarity coefficients $C=(72.2 \pm 0.1) \times 10^{-4} \mu \mathrm{m}^{4} / \mathrm{cm}$ and $C=(64.4 \pm 0.1) \times 10^{-4} \mu \mathrm{m}^{4} / \mathrm{cm}$ were determined for each tile fitting equation (1). The clarity determined for the $8 \mathrm{~cm}$ stack was $C=(69.5 \pm 0.1) \times 10^{-4} \mu \mathrm{m}^{4} / \mathrm{cm}$. The $A$ coefficients were $(92.0 \pm 0.1) \%$ and $(95.9 \pm 0.1) \%$ for the individual tiles and $(88.2 \pm 0.1) \%$ for the $8 \mathrm{~cm}$ thickness, well in agreement with the multiplicative law of $A_{i}$.

Tiles produced by Matsushita [2], which are hydrophobic, were also tested. A large tile of $20 \times 30 \times 2 \mathrm{~cm}^{3}$ with a clarity coefficient $C=(100.3 \pm 0.2) \times 10^{-4} \mu \mathrm{m}^{4} / \mathrm{cm}$ and 
$A=(82.0 \pm 0.1) \%$, was cut into four tiles, so that thicknesses of 2 to $8 \mathrm{~cm}$ could be obtained. The nominal refractive index was 1.030 at wavelength $\lambda=543.5 \mathrm{~nm}$.

The light scattering length in aerogels produced by Matsushita [2] and Boreskov Institute of Catalysis [3] are unequal due to wide differences in the production procedure and raw materials used, which result in different structure of aerogel (hydrophobic and hygroscopic aerogels).

Data were taken under different conditions, changing the thickness of the aerogel (4, 6 and $8 \mathrm{~cm})$, at different beam momenta $(6,8,9,10 \mathrm{GeV} / \mathrm{c})$ and particle charge. Highly relativistic charged particles $(\beta \simeq 1)$ produce Cherenkov photons in the aerogel with refractive index $n=1.030$ at an angle of $242 \mathrm{mrad}$ with respect to the particle direction. Different kinds of filters were added in some runs, at the exit wall of the aerogel radiatior, in order to absorb UV photons above $\sim 3 \mathrm{eV}$, which are most affected by Rayleigh scattering.

\section{Experimental set-up}

The set-up is shown in Fig. 2 and consists of a light-tight stainless-steel cylindrical vessel with a diameter of $50 \mathrm{~cm}$ and a length of $108 \mathrm{~cm}$. It houses the aerogel radiator, a focusing mirror and four photodetectors. Nitrogen was flushed through the vessel to protect hygroscopic aerogel from humidity.

\subsection{The Beam}

In the East Hall experimental area at CERN, a primary proton beam of $24 \mathrm{GeV} / \mathrm{c}$ is extracted from the PS synchrotron. For each PS supercycle $(14.4 \mathrm{~s})$ the beam comes in 2 spills of $300 \mathrm{~ms}$, providing $2 \times 10^{11}$ particles on the 2 downstream targets.

The secondary beams can be selected in momentum and charge. For the test described in this paper, the $\mathrm{T} 7$ secondary beamline was used, providing up to $10^{6}$ secondaries per spill, with a maximum momentum of $10 \mathrm{GeV} / \mathrm{c}$ and $1 \%$ uncertainty.

The beamline can yield a pure $\pi^{-}$beam, or a mixture of $\pi^{+}$and $p$, the proton content increasing from $50 \%$ at $6 \mathrm{GeV} / \mathrm{c}$ up to $70 \%$ at $10 \mathrm{GeV} / \mathrm{c}$.

In order to determine the direction and position of the incoming particles, two silicon pad sensors (484 pads of $1.3 \times 1.3 \mathrm{~mm}^{2}$ ), were located upstream and one downstream the vessel, to define the position and direction of the incoming particle impinging on the aerogel radiator, allowing for a spatial resolution of about $1 \mathrm{~mm}$.

During the test, the beam was defocused to reduce the pile-up probability and the last dipole magnets were adjusted in order to have the beam centered on the 3 silicon planes.

\subsection{The Photodetectors}

The focal plane of the set-up was equipped with four Hybrid Photon Detectors (HPD), developed and fabricated at CERN. The basic design, performance and fabrication of the Pad HPD is described in $[9,10]$. We summarize here only the main characteristics.

The Pad HPD is a round hybrid photodiode of $127 \mathrm{~mm}$ diameter with a bialkali photocathode of $114 \mathrm{~mm}$ active diameter (see Fig. 3). The spherical entrance window of the HPD is made of an UV extended borosilicate glass $(\mathrm{T}=50 \%$ at $\lambda=250 \mathrm{~nm})$. Compared to 
the original design, the $2^{\text {nd }}$ generation Pad HPD used in this experiment, has a simplified electrode structure. Two concentric ring electrodes plus a wire electrode of $1 \mathrm{~mm}$ diameter define a fountain shaped electric field, which demagnifies the photocathode image by a factor $\sim 2.3$ onto a silicon sensor of $50 \mathrm{~mm}$ diameter. The silicon sensor comprises 2048 pads of size $1 \times 1 \mathrm{~mm}^{2}$. In addition to the advantages in the tube manufacturing, the new electrode structure improves the operational stability. Each HPDs can be operated at a cathode voltage of $20 \mathrm{kV}$. For this experiment all 4 HPDs were supplied in parallel by a single set of high voltage power supplies ${ }^{2}$. Imperfections in the positioning of the bleeder electrode with respect to the cathode surface lead for some of the tubes to cross focusing effects in the peripheric region $\left(R_{\text {cathode }} \geq 45 \mathrm{~mm}\right)$ as shown in Fig. 4 . These imaging distortions can be reduced by stricter geometrical tolerances or by supplying each tube individually with an optimized set of voltages. For the detection of very low light levels, as expected for an aerogel radiator ( $<1$ p.e. $/ \mathrm{HPD})$, a cathode voltage of $16 \mathrm{kV}$ represented the best compromise between detection efficiency and random 'dark' noise. Under these conditions a random hit probability of $7 \times 10^{-4}$ per pad and per trigger was found while the single photoelectron detection efficiency was estimated to be as high as $94 \%$ for a $4 \sigma$ pedestal cut of the analogue signal. The point spread error at $16 \mathrm{KV}$ is about $0.5 \mathrm{~mm}$ on the Si sensor. The quantum efficiency (QE) of the 4 HPDs, measured at normal incidence in the center of the cathode, is shown in Fig. 5. All detectors had their maximum QE in the range from $20 \%$ to $30 \%$. The cut-off for the short wavelengths is due to the borosilicate window.

\subsection{The Mirror}

A mirror of radius of curvature $\mathrm{R}=118.5 \mathrm{~cm}$ was mounted such that the focal plane coincided with the entrance windows of the four HPDs. The mirror reflectivity is shown in Fig. 6. The average geometric precision of the mirror corresponds to an angular dispersion of about $0.5 \mathrm{mrad}$. This gives a negligible contribution to the angular resolution. The distance between the centers of the HPD photocathodes and the geometrical center of the end plate was $14.6 \mathrm{~cm}$. The alignement was performed among the optical elements with a laser to a precision of $1.5 \mathrm{mrad}$.

\section{Data Acquisition}

The front-end electronics for the HPDs are encapsulated within the vacuum envelope of the tube. The function of the front-end electronics is to capture the analogue signals simultaneously from all of the 2048 pads and to multiplex the analogue data onto a single analogue output. The required functions are available in a number of different ASICs from which the VA2 was chosen as it has excellent signal-to-noise characteristics and is relatively straightforward to operate.

Each VA2 ASIC preamplifies and shapes the analogue signals from 128 inputs simultaneously. The characteristic rise time of the shaped signal is around $1.3 \mu \mathrm{s}$. The total time needed to multiplex out 2048 signals at $5 \mathrm{MHz}$ is around $0.5 \mathrm{~ms}$ during which time the front-end cannot capture a new event.

\footnotetext{
${ }^{2}$ Ultra low ripple power supplies, http://www.matsusada.com.
} 
In these measurements, the four HPDs delivered 4 streams of multiplexed analogue signals. The analogue data were digitised using VME CRAMS modules.

The CRAMS modules are capable of sampling a continuous stream of analogue data up to a maximum of 2032 samples. This is slightly less than the 2048 signals from one HPD and results in the last few signals being lost. The CRAMS can perform pedestal subtraction and zero-suppression of the data in hardware and this feature was exploited to reduce the volume of data recorded per event. The trigger was formed using external NIM logic from the coincidence of 4 beam scintillators.

At the end of the readout sequence for each triggered event the digitised, zero-suppressed event data from the HPDs remains in the CRAMS memory until they are copied by the data acquisition software into the memory of the VME single board computer that acts as the DAQ controller. At the end of each PS cycle, the buffered data are then moved onto permanent storage via a $100 \mathrm{Mbit} / \mathrm{s}$ Ethernet LAN.

The silicon telescope front-end electronics is also based on the VA series ASIC and so the same readout system was used to acquire both HPD and telescope data.

For the online monitoring of the HPD data and the beam-telescope, a monitoring program has been developed. It reads the event data and fills histograms. A scripting language is used to provide a flexible graphic display of the event data.

\section{Monte Carlo Simulation}

A detailed simulation program has been developed using the GEANT4 software toolkit [11]. This program configures the geometry of the experimental set-up and simulates the various physics processes.

In the program, a beam of charged pions going through the aerogel and the nitrogen produces Cherenkov photons uniformly in energy and with the corresponding Cherenkov angles along their trajectory. The chromatic aberration was determined using the Sellmeier dependence of the refractive index shown in Fig. 7 as a function of the photon wavelength. This behaviour was found to be in agreement with the experimental measurements from ref. [16]. If the dependance is assumed to be $20 \%$ larger, the resulting chromatic error would be correspondingly $20 \%$ worse.

The photons incident on an optical boundary undergo reflection and refraction according to Fresnel equations [8]. The external boundaries of the aerogel and the filter are examples of the optical boundaries in this set-up. In Fig. 8 the transmission of the filters used [12], measured with a spectrophotometer, is shown. When a filter was used, these curves were used in the simulation.

The photons travelling in the aerogel undergo Rayleigh scattering [8] and absorption in such a way that the transmission $\mathrm{T}$ of photons is described by eq.(1). The reflectivity of the mirror used in the simulation is the measured one (c.f. Fig. 6).

A fraction of the photons is not converted to photoelectrons, but is transmitted through the HPD window and reflected from the aluminized silicon surface back to the photocathode, and only then the conversion occurs. A correction was applied to the QE used in the simulation to account for this effect of transparency of the photocathode in about $20 \%$ of photons traversing it at a distance $\geq 25 \mathrm{~mm}$ from the tube axis.

The photoelectrons are assigned the energy corresponding to the electric potential between the anode and cathode of the HPD and their impact position on the silicon anode 
are set by the fountain focusing law and the point spread function indicated in Section 3.2 of this paper. There is $\sim 18 \%$ probability [13] at normal incidence for electrons to backscatter at the silicon detector surface with a flat distribution of the energy lost in the sensor. These electrons are expected to be detected only if they impart an energy to the silicon above the detection threshold. Therefore, to simulate this effect, a hit detection efficiency $\epsilon$ is applied to the photoelectrons according to the formula: $\epsilon=1-B \cdot n_{\sigma} / S N R$ where $B$ is the backscattering probability, $n_{\sigma}$ is the detection threshold in units of standard deviations of the pedestal distribution and $S N R$ is the signal to noise ratio.

The photoelectrons passing this efficiency criterium are assumed to create signal in the silicon pads.

\section{Experimental Results}

For each configuration (aerogel thickness, beam energy, filter or no filter behind the aerogel), a statistics of about 30,000 events was taken.

In order to study the background and the noise of the detectors, runs were taken with the trigger set out of the beam spill, after each data run. The number of hits per event,

$N_{b c k g r}$, was calculated in these runs in the same way as for normal runs and was used to evaluate the background to be subtracted in each pad.

Pads counting more than $2 \times 10^{-3}$ hits per event, were classified as noisy and excluded from the analysis. The number of noisy pads which were masked was between 10 and 40 per HPD. Two full sectors (128 pads each) were found noisy in HPD2 and masked. Pads were defined inefficient if they had no hits in both the data and the background runs. With an average number of 30,000 events per run, the probability for an efficient pad in the Cherenkov ring region to collect zero events is negligible.

\subsection{Data Analysis}

At the acquisition level, a cut at $4 \sigma$ above the pedestal was applied, which corresponds to about 12 ADC counts.

The position of the single photoelectron peak was found to be around 30 ADC counts, with a global pad to pad variation of about $4 \%$, which reflects the dispersion of the frontend electronics gain, hence a variation inside the same sector of about $2.5 \%$ and a maximum variation between different sectors of $10 \%$. An example of signal spectra is shown in Fig. 9. To select the region with the Cherenkov signal, a cut on the ADC counts was applied. In the case of photoelectron counting the amount of signal lost below this cut was calculated and corrected for (see Section 6.2).

For the Cherenkov angle reconstruction the effective position and orientation of the 4 HPDs on the endplate of the vessel was found by a software geometrical re-alignment. A correction for the relative position of the 4 HPDs was applied to the data, to correct $a$ posteriori an observed misalignement.

An example of ring reconstruction is given in Fig. 10 where the result of the fit to the observed 2-dimensional hit distribution, after noise subtraction, is obtained by minimisation of the quantity,

$$
S=\sum_{i}\left[\left(x_{i}-x_{0}\right)^{2}+\left(y_{i}-y_{0}\right)^{2}-R^{2}\right]^{2}
$$


where $x_{0}, y_{0}$ are the coordinates of the center of the ring, $R$ is the radius and the sum is over all hits. The minimum of $S$ can be found analytically, as described in [14], obtaining the radius and its error, when the number of photoelectrons in an event is larger than 3. For all the results described in this paper, the Cherenkov emission angle $\theta_{c}$ is extracted from the photoelectron hit coordinates and the complete knowledge of the geometry of the system using the retracking algorithm described in [15].

\subsection{Photoelectron Yield}

The photoelectron yield is defined as the average number of photoelectrons detected per event. The number of photoelectrons has been evaluated separately in each HPD, summing over all the pads in the geometrical region defined as three times the ring radius $(R)$ resolution $\left( \pm 3 \sigma_{R}\right)$ around the fitted $R$ ('on-ring' region), or in the complementary region ('off-ring' region).

A radial cut has been applied in order to eliminate the region of the silicon anode being affected by cross-focusing effects, which may give rise to reconstruction ambiguities (see Fig. 4). The extension of this region is different for the four HPDs, but for simplicity an active diameter of $74 \mathrm{~mm}$ has been used for all of them. With this restriction, the fraction of Cherenkov ring area covered by the four HPDs corresponds to about $32 \%$ of the entire ring.

The number of photoelectrons in each pad has been calculated analysing the corresponding ADC spectra. Since the average number of photoelectrons per event on a single pad is smaller than 0.01 , the probability of more than one photoelectron per pad is negligible. Therefore the integrals of the measured ADC spectra correspond to the sum of single photoelectron hits and background.

The position of the single photoelectron peak was determined from a Gaussian fit to the ADC spectra in each $\mathrm{HPD}$, in the range from $-1.5 \sigma$ to $+4 \sigma$ in order to minimize the contribution of the backscattering. The number of hits per event, $N_{\text {hits }}$, was thus calculated summing hits between $-3 \sigma$ and $4 \sigma$ from the mean value. In HPD2, due to a lower gain, the lower threshold was fixed at $2 \sigma$ from the mean value.

The average number of photoelectrons per event is finally obtained taking the difference of the results of a Poisson fit to the distributions of $N_{\text {hits }}$ and $N_{b c k g r}$. The following corrections have been applied:

- The photoelectron losses due to masked or inefficient pads. This correction was determined from the simulation for each HPD to vary from $2 \%$ to $15 \%$ in the ring region and from $2 \%$ to $25 \%$ in the off-ring region.

- The signal loss below threshold in HPD2 (2\%)

- The signal loss below threshold, due to backscattering $(14.2 \pm 0.2) \%$.

- The azimuthal acceptance of the photodetectors (32\%).

The same procedure has been applied to the MonteCarlo simulation. The comparison between data and MonteCarlo, for different thicknesses of aerogel and filter, are shown in Table 1 and Table 2. The extrapolation is made to the full plane, not considering the unavoidable packing factor and the outer photondetector dimension, which will limit the full acceptance. 
The photoelectron yields of the individual HPDs are in good agreement with their quantum efficiency characteristics (c.f. Fig. 5).

\begin{tabular}{|rc|c|c|}
\hline \multicolumn{2}{|c|}{ on-ring } & No Filter & Filter D263 \\
\hline $4 \mathrm{~cm}$ & Data & $9.7 \pm 1.0$ & $6.3 \pm 0.7$ \\
& MC & $11.5 \pm 1.2$ & $7.4 \pm 0.8$ \\
\hline $8 \mathrm{~cm}$ & Data & $12.2 \pm 1.3$ & $9.4 \pm 1.0$ \\
& MC & $14.7 \pm 1.6$ & $10.1 \pm 1.1$ \\
\hline
\end{tabular}

Table 1: Photoelectron yield, in the 'on-ring' region, normalised to the full $2 \pi$ acceptance when using the Novosibirsk aerogel type in runs with and without the filter.

\begin{tabular}{|cc|c|c|}
\hline \multicolumn{2}{|c|}{ off-ring } & No Filter & Filter D263 \\
\hline $4 \mathrm{~cm}$ & Data & $1.13 \pm 0.21$ & $0.67 \pm 0.11$ \\
& MC & $0.87 \pm 0.09$ & $0.55 \pm 0.06$ \\
\hline $8 \mathrm{~cm}$ & Data & $1.38 \pm 0.23$ & $1.25 \pm 0.21$ \\
& MC & $1.34 \pm 0.15$ & $0.94 \pm 0.10$ \\
\hline
\end{tabular}

Table 2: Photoelectron yield, in the 'off-ring' region, for the Novosibirsk aerogel. In this case the numbers are expressed in units of $10^{-2} / \mathrm{cm}^{2}$.

The statistical error on the number of photoelectrons is below $1 \%$. The systematic error on the number of photoelectrons in the data, per HPD, includes the contribution from the following sources:

- Signal losses outside ADC thresholds: $2 \%$

- Background subtraction: $1 \%$ to $8 \%$ in the ring region and $6 \%$ to $15 \%$ in the off-ring region, respectively, depending on the HPD.

- Inefficient or noisy pads: $2 \%$ to $7 \%$ in the ring region and $2 \%$ to $16 \%$ in the off-ring region, respectively, depending on the HPD.

- Definition of "on-ring" and "off-ring" boundaries: 5\% (ring), 7\% (off-ring)

- Extrapolation to $2 \pi$ : $5 \%$

For the simulation the systematic error in the photoelectron yield is obtained by varying

- the QE of the phototube by $10 \%$ at all wavelengths ( $\pm 10 \%$ on the p.e. yield),

- the refractive index of the aerogel $(n-1)$ by $5 \%$ at all wavelengths $( \pm 3 \%$ on p.e. yield),

- the clarity of aerogel by $2 \%$ ( $\pm 2 \%$ on p.e. yield $)$,

- the beam direction by $1 \mathrm{mrad}$ in different directions ( $\pm 1 \%$ on p.e. yield), 
- and the backscattering probability in silicon between $16 \%$ and $20 \%$ ( $\pm 2 \%$ variation on p.e. yield).

\subsection{Cherenkov Angle Reconstruction and Resolution}

The Cherenkov emission angle $\theta_{c}$ is extracted from the photoelectron hit coordinates and the complete knowledge of the geometry of the system using the retracking algorithm described in [15].

The solid lines in Fig.11 show the measured $\theta_{c}$ distribution of single photoelectrons for a run of about 30k events taken with $4 \mathrm{~cm}$ Novosibirsk type aerogel and with and without the glass filter type D263. The MC simulation is superimposed as dashed line.

The shoulder right of the peak $\left(\theta_{C} \sim 0.26-0.28 \mathrm{rad}\right)$ is due to the photons which traverse the semi-transparent photocathode, bounce back from the aluminized silicon sensor and are only then converted to photoelectrons, as described already in section 5, and appear as a shadow to the main ring in Figure 10. In the MonteCarlo, this second ring is clearly visible in fig.11(right), due to the better resolution.

In Fig. 12 a direct comparison of the two types of aerogel samples is shown. The worse resolution of the Matsushita sample, visible especially in the left figure (data without filter), and given in tables 3 and 4 , is coming most probably from the different optical quality, which, over $8 \mathrm{~cm}$ thickness, diffuse in a more relevant way.

In all set-up configurations quality cuts were applied to the ADC distributions of the 4 HPDs in order to increase the signal/background ratio. Pads with an occupancy above $2 \%$ were excluded from the analysis.

The Cherenkov angle has been corrected for the refraction at the exit of the aerogel, assuming that the frontal surface of the aerogel tile was parallel to the detectors plane.

Table 3 shows the measured Cherenkov mean angle $\theta_{C}$ and the single photoelectron resolution $\sigma_{\theta}$ corresponding to the two filters used, the type of the aerogel and its thickness. All the photons within the cuts defined before and in all the events, have been used. The measured values are compared to Monte Carlo predictions. It must be noticed that the indeces of refraction of the different samples tested are slightly different, being, when compared at $400 \mathrm{~nm}, 1.0306,1.0302,1.0309$, for the samples Novosibirsk $4 \mathrm{~cm}, 8 \mathrm{~cm}$, and Matsushita respectively.

The angular resolution was determined from a fit using a Gaussian plus a linear contribution. The resolutions $\sigma_{\theta}$ quoted in Table 3 refer only to the Gaussian part of the distributions. Table 4 shows the ratio of the gaussian contribution to the total area.

The resolution $\sigma_{\theta}$ listed in Table 3 for the Monte Carlo simulations corresponds to the contributions from the sources listed in Table 5. The largest contribution comes from the chromaticity of the emitted photons, enhanced by the Rayleigh scattering.

The measured Cherenkov angle resolution depends upon the number $N_{\text {p.e. }}$ of detected photoelectrons as

$$
\sigma_{\theta}^{r}=\frac{\sigma_{\theta}}{\sqrt{N_{p . e .}}} \oplus k
$$

where $\sigma_{\theta}$ is the single photoelectron resolution, and $k$ accounts for possible systematic errors which do not scale with the number of photoelectrons, and were not described in the MonteCarlo.

Fig. 14 shows the measured dependence of the resolution with the number of photoelectrons. It is obtained subdividing the events according to their $N_{\text {p.e. }}$ multiplicity. Fit- 


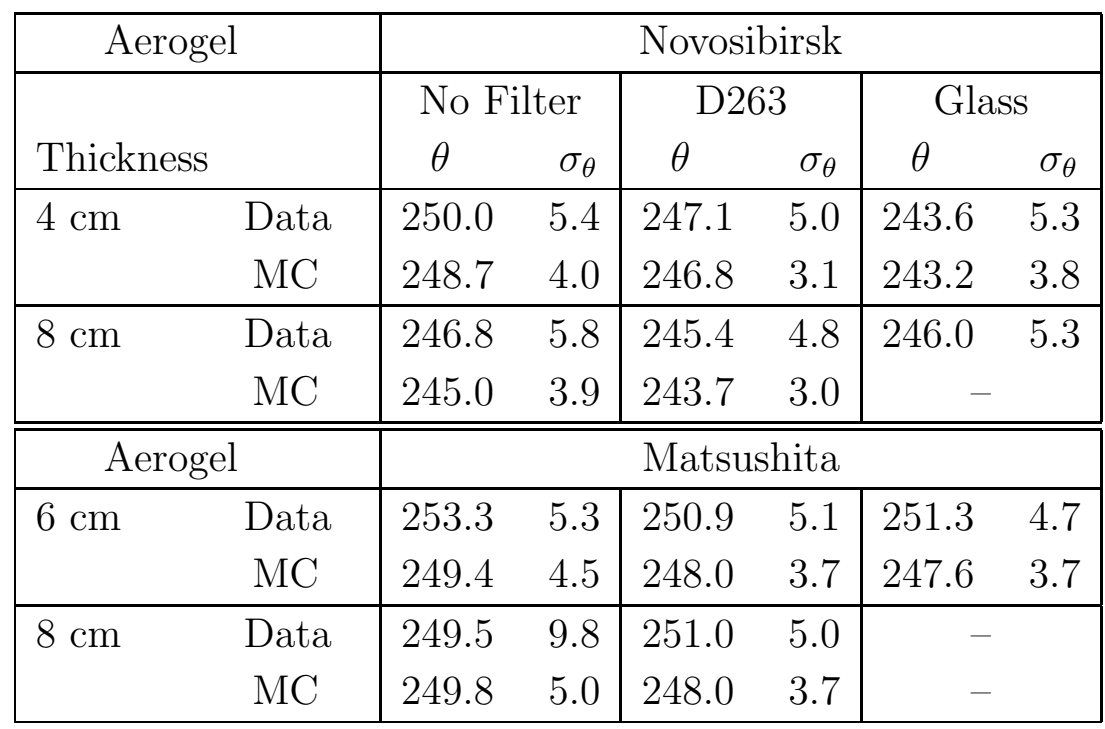

Table 3: Cherenkov emission mean angles and single photoelectron resolutions in mrad for different aerogel types and thicknesses, and for the different filters used.

\begin{tabular}{|cl|c|c|c|}
\hline & & No Filter & D263 & Glass \\
\hline Novosibirsk & $4 \mathrm{~cm}$ & 0.53 & 0.58 & 0.55 \\
& $8 \mathrm{~cm}$ & 0.56 & 0.52 & 0.52 \\
\hline Matsushita & $6 \mathrm{~cm}$ & 0.39 & 0.45 & 0.26 \\
& $8 \mathrm{~cm}$ & 0.47 & 0.33 & - \\
\hline
\end{tabular}

Table 4: Ratio between the Gaussian contribution in the fit and the total area.

ting the data with Eq. 3, gives the values $\sigma_{\theta}=4.8 \pm 0.3 \mathrm{mrad}$ and $k=2.0 \pm 0.1 \mathrm{mrad}$. The same treatment applied to the MonteCarlo events, yields the values $\sigma_{\theta}=3.2 \pm 0.2 \mathrm{mrad}$ and $k$ compatible with 0 mrad. A critical comparison data-MonteCarlo is discussed in section 6.5.

\section{$6.4 \pi / \mathrm{p}$ Separation}

While in runs with negative beam, only $\pi^{-}$are present, in positive runs the beam is composed of $\pi^{+}$and protons, giving rise to two separate Cherenkov rings. Fig. 13 shows the Cherenkov angle distributions for single photoelectron, after noise subtraction, in runs with the beam momentum set at $+6 \mathrm{GeV} / \mathrm{c},+8 \mathrm{GeV} / \mathrm{c}$ and $+10 \mathrm{GeV} /$ c respectively.

The angular separation of the pion and proton rings $\Delta \theta=\theta_{\pi}-\theta_{p}$ is usually expressed in terms of standard deviations: $N_{\sigma}=\Delta \theta / \sigma_{\pi}$. The number of detected photoelectrons is extrapolated to full azimuthal coverage. Table 6 shows the results for different beam momenta in the case of $4 \mathrm{~cm}$ Novosibirsk aerogel with the D263 filter type. The resolution of the proton angle is probably degraded by an effect of distorsion at the phototubes periphery: decreasing the proton momentum, the ring is located closer and closer to the border of the anode.

From Table 6, a good separation power of the aerogel can be observed in the en- 


\begin{tabular}{|c|c|}
\hline Source & $\sigma(\mathrm{mrad})$ \\
\hline \hline Pixelling & 1.3 \\
\hline Chromaticity & 2.5 \\
\hline $\begin{array}{c}\text { Point Spread fnc } \\
+ \text { Emission point }\end{array}$ & 1.1 \\
\hline Beam divergence & 0.7 \\
\hline \hline Total & 3.1 \\
\hline
\end{tabular}

Table 5: Contributions to the single photoelectron resolution in the MC simulation for runs with $4 \mathrm{~cm}$ aerogel Novosibirsk aerogel and filter D263.

\begin{tabular}{|c|cc|cc||c|}
\hline Particle Momentum & $\theta_{p}$ & $\sigma_{p}^{r}$ & $\theta_{\pi}$ & $\sigma_{\pi}^{r}$ & $N_{\sigma}$ \\
\hline \hline$+6 \mathrm{GeV} / \mathrm{c}$ & 194.0 & 7.8 & 243.6 & 2.9 & 17.1 \\
\hline$+8 \mathrm{GeV} / \mathrm{c}$ & 216.4 & 4.1 & 244.3 & 2.8 & 9.9 \\
\hline$+10 \mathrm{GeV} / \mathrm{c}$ & 224.8 & 3.0 & 242.8 & 2.3 & 7.8 \\
\hline
\end{tabular}

Table 6: Reconstructed Cherenkov angles and resolutions in mrad for a mixed $\pi^{+} / p$ beam at various energies. and with $4 \mathrm{~cm}$ Novosibirsk aerogel with the D263 filter type. The resolutions quoted correspond to the $\mathrm{N}_{p . e \text {. }}$ extrapolated to the full azimuthal coverage.

ergy range $6-10 \mathrm{GeV}$, between positive pions and protons. For the $K / \pi$ separation, the numbers would correspond to $\mathrm{N}_{\sigma}=4.7,2.9,1.6$.

\subsection{Comparison Data/MonteCarlo}

In general, the Monte Carlo simulation shows better results compared to the data. While the $N_{\text {p.e. }}$ yield agrees within $\sim 15 \%$, the angular resolution (c.f. Table 3 ) turns out to be better by about $30 \%$ in the MonteCarlo $(3.1 \mathrm{mrad})$ than in the data $(4.8 \mathrm{mrad})$. This implies that one or more contributions are not well simulated or are missing in the MonteCarlo description.

Effects due to forward scattering in the aerogel [16], imperfections of the mirror geometry, are degrading the final resolution. Effects due to misalignement, optical distorsion in the HPDs are eventually also adding contributions to the resolution. For example, the same power supply distributed the same fields to all the 4 HPDs, therefore they were not optimized for each of them.

To investigate if a possible reason for the worse angular resolution could be due to an inhomogeneity of the HPDs response, as Fig. 10 and 13 suggest, a direct comparison of the mean Cherenkov angle and its resolution is done in different regions of the anode and for each HPD. The angle $\varphi$ in Fig. 15, is defined as the difference of the azimuthal angle of the emitted photon and the azimuthal angle of the center of the considered HPD. For five angular regions and for each HPD, the mean value of the Cherenkov angle (bottom) and its resolution (top) are plotted. From the figure, it can be noticed that there are two sources which worsen the final resolution quoted in Table 3. The first is a spread of the 
mean Cherenkov angle (up to $4 \mathrm{mrad}$ ), the second is a degradation of the resolution at the anode periphery.

A dedicated testbeam must be made in the future, with optimal conditions for the photodetectors, the optical system, the alignement, in order to investigate deeper the discrepancy with the MonteCarlo simulation.

\section{Conclusion}

The excellent quality of the aerogel available from Novosibirsk makes it a good Cherenkov radiator and encourages its use in a RICH detector in a high multiplicity environment, as it is the case for $p-p$ interactions. The ring image has the requisite number of photoelectrons.

From the data presented in this paper, a thickness of $8 \mathrm{~cm}$ is preferred for the better photoelectron yield, with no significant difference in the angular resolution, compared to the results from a $4 \mathrm{~cm}$ thickness. The effect of the filters, aiming at reducing the chromatic error, is visible in the MonteCarlo and in the data, where however there are extra contributions not described by the simulation.

The angular resolution allows a good separation between pions and protons, but a better understanding of the different contributions and a better description by the MonteCarlo is mandatory.

\section{Acknowledgments}

We would like to thank T. Duane and R. Mazza for their competent help. We would like to thank C. David, R. Dye, M. Malabaila and M. Thiebert (CERN) for their valuable support in the preparation of the HPD components. The careful mounting and bonding of the sensors and the chip sets by B. Herskind (CERN) is acknowledged. We appreciate the financial support of part of this work by INFN Bologna.

We acknowledge the financial support by INTAS contract 679 .

\section{References}

[1] R. De Leo et al, NIM A401 (1997) 187

[2] H.Yokogawa and M.Yokoyama, Journal of Non-Crystalline Solids 186(1995)23-29

[3] A.F.Danilyuk et al., Nucl.Inst.Meth. A433 (1999) 406.

[4] HERMES Collaboration, K. Ackerstaff et al., Nucl.Inst.Meth. A417 (1998) 230.

[5] LHCb TDR 3, CERN/LHCC/2000-0037, september 2000.

[6] AMS Collaboration, M. Aguilar et al., Physics Reports 366 (2002) 331.

[7] M. Calvi et al., ICHEP 2001, Budapest, Hungary Jour. High En. Phys., Conf. Proc, PRHEP-hep2001/271.

[8] Classical Electrodynamics, J.D. Jackson, John Wiley \& Sons Inc. NewYork, 1975. ISBN 0-471-43132-X. 
[9] A. Braem et al., Nucl. Instr. Meth. A442 (2000) 128

[10] A. Braem et al., Nucl. Instr. Meth. A478, (2002) 400

[11] GEANT4 Status and results, J.Apostolakis et al., Proceedings of the CHEP2000 conference.

[12] The filters used were ordinary glass and thin glass D263 available from DESAG, D-31074 Gruenenplan.

[13] E. Darlington, J. Phys D8 (1975) 85.

[14] J.F. Crawford Nucl. Instr. Meth. 211 (1983) 223.

[15] R. Forty, NIM A384 (1996) 167.

[16] R. De Leo et al., NIM A457 (2001) 52.

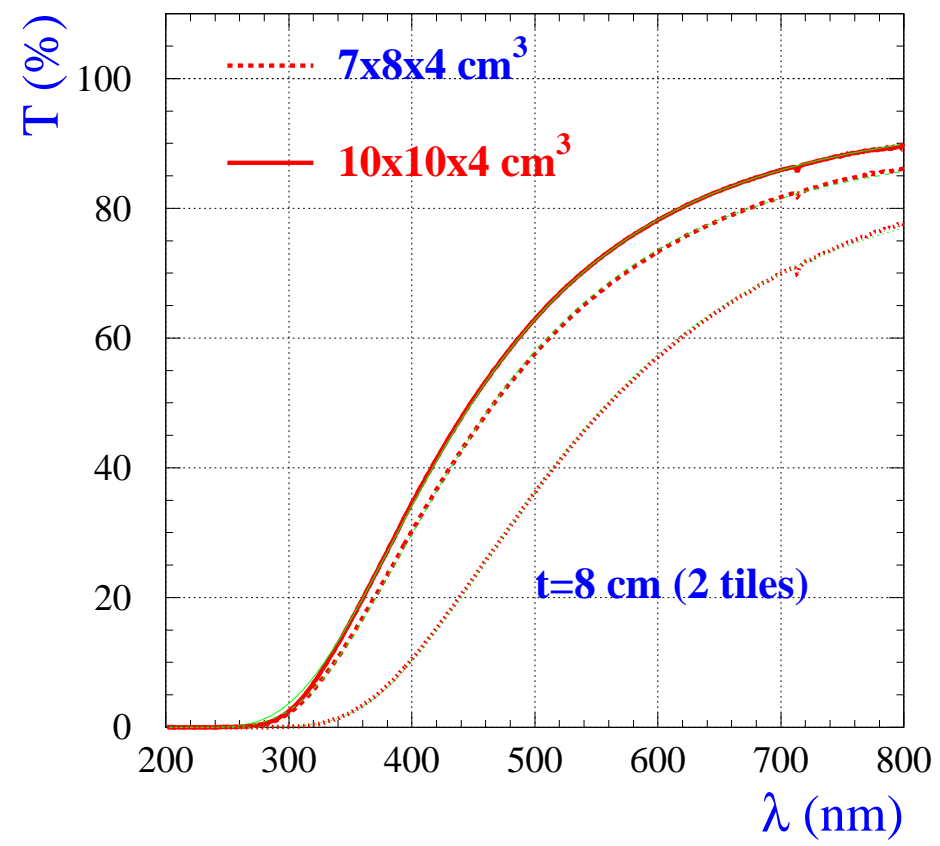

Figure 1: The transmittance as a function of wavelength for two samples $4 \mathrm{~cm}$ thick, produced in Novosibirsk (solid and dashed lines), and for the two samples together (dotted line) 


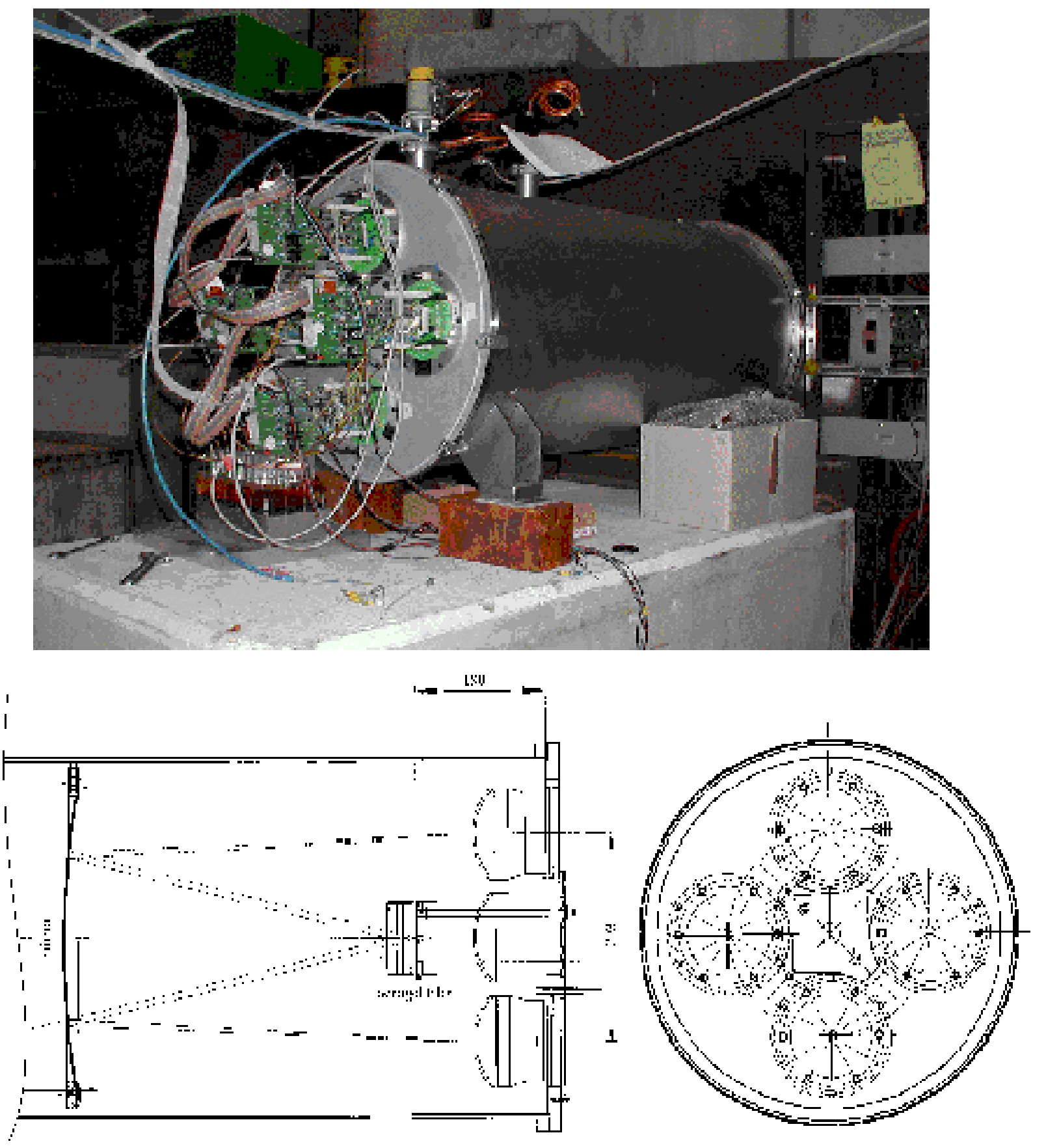

Figure 2: The experimental set-up used for the test. Top: a picture of the apparatus. Bottom: a sketch of the vessel and the end plate housing the 4 HPDs 


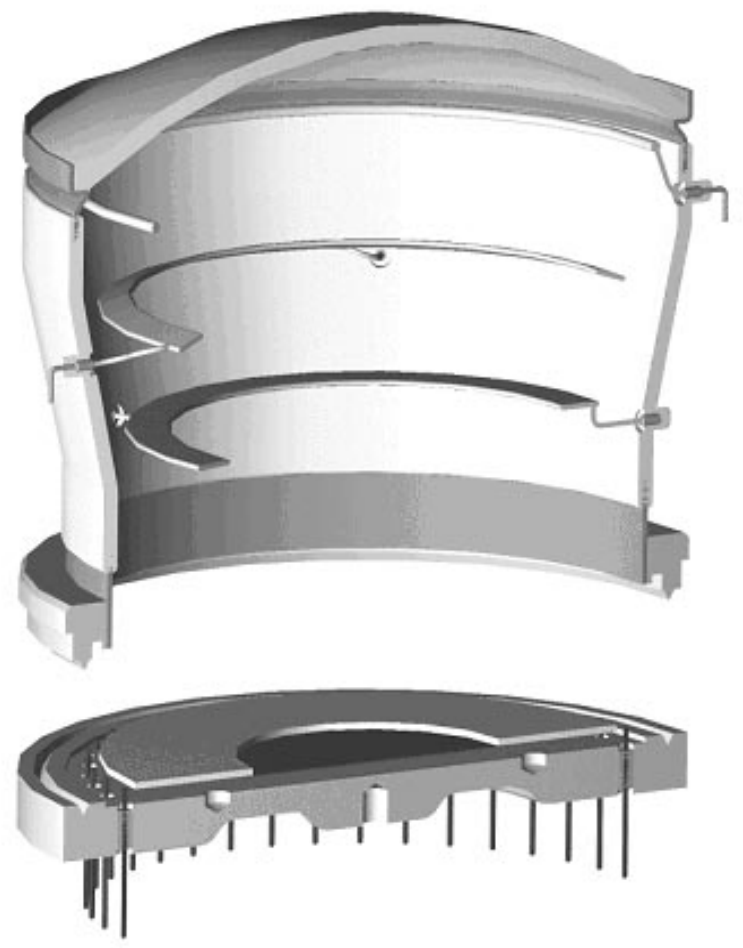

Figure 3: 3D view of the Pad HPD with second generation envelope. 


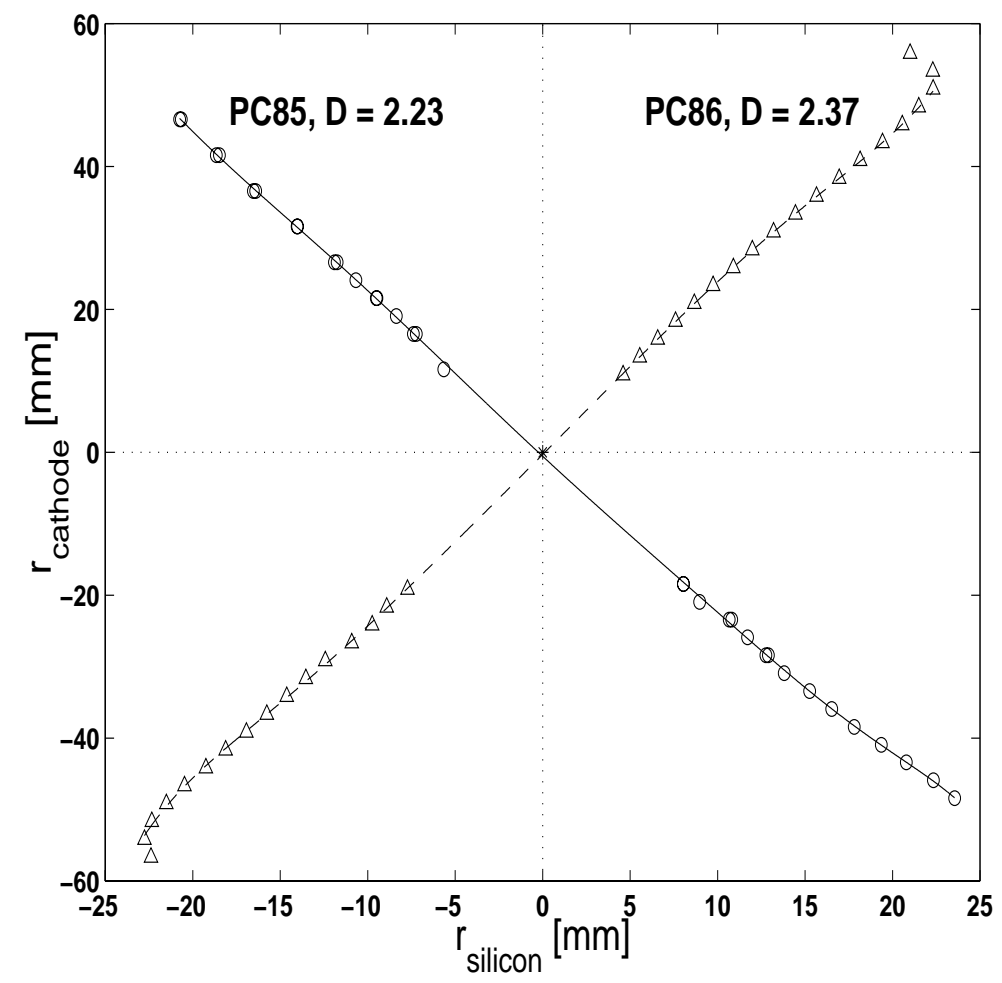

Figure 4: Demagnification function of HPD-2 and HPD-4 (corresponding to the photocathodes PC85 and PC86 respectively). The plot shows the result of the radial scan of a focused light source over the cathode surface. HPD-4 shows slight cross-focusing effects at large radius. The demagnification $\mathrm{D}$ is measured in the centre of the HPDs.

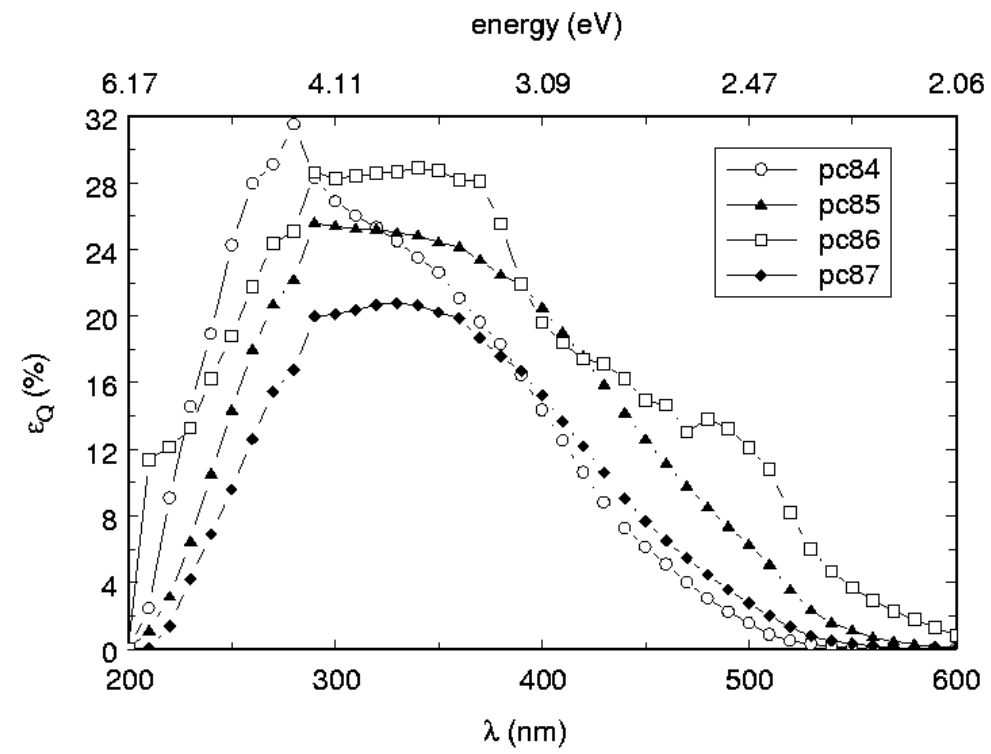

Figure 5: Quantum efficiency of the 4 HPDs used in the experiment. 


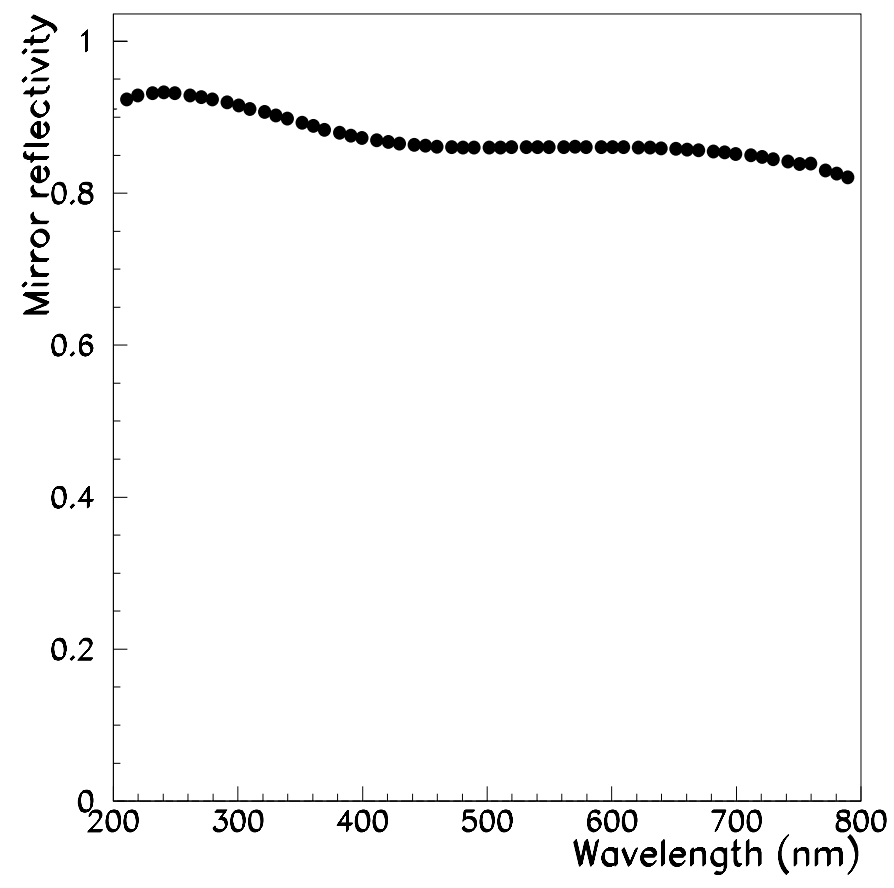

Figure 6: Reflectivity of the mirror as a function of the photon wavelength in $\mathrm{nm}$.

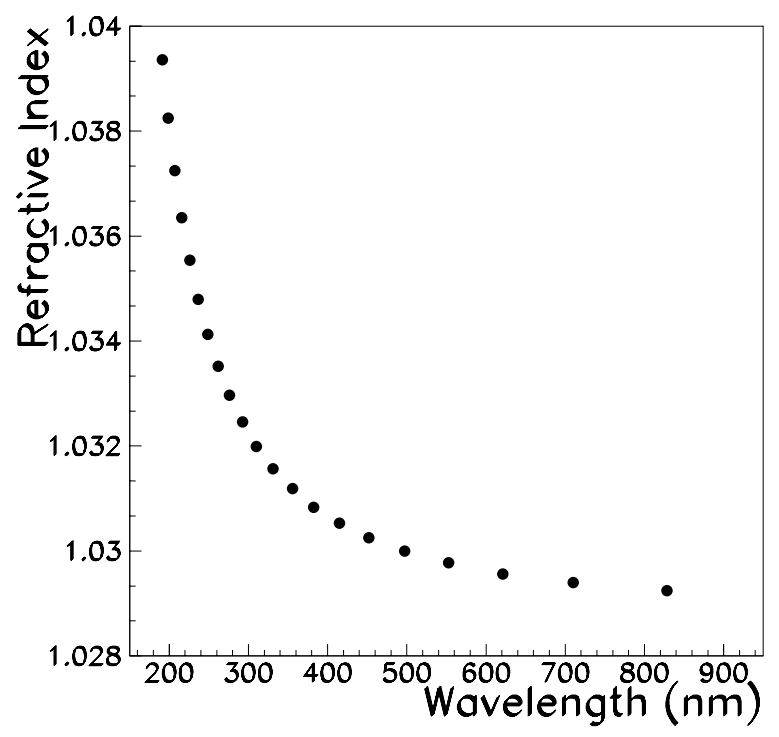

Figure 7: Refractive index as calculated by the MonteCarlo, of an aerogel tile as a function of the photon wavelength in $\mathrm{nm}$. 


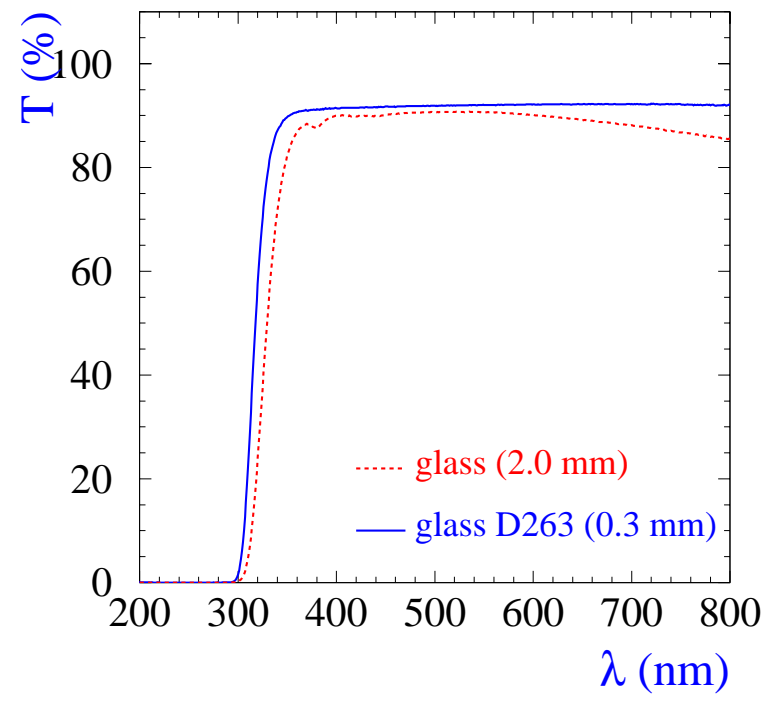

Figure 8: Transmission of the optical filters as a function of the photon wavelength in nm.

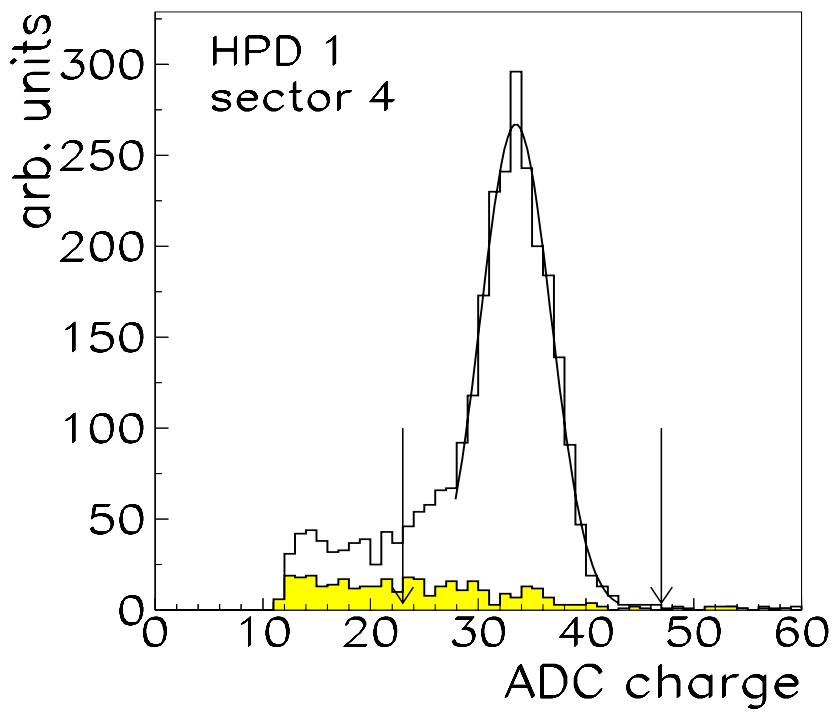

Figure 9: The ADC charge spectra summed over 128 pads (one HPD sector) for $4 \mathrm{~cm}$ of aerogel. The arrows show the thresholds defining the accepted events. The shaded area corresponds to data taken in the "background run". 


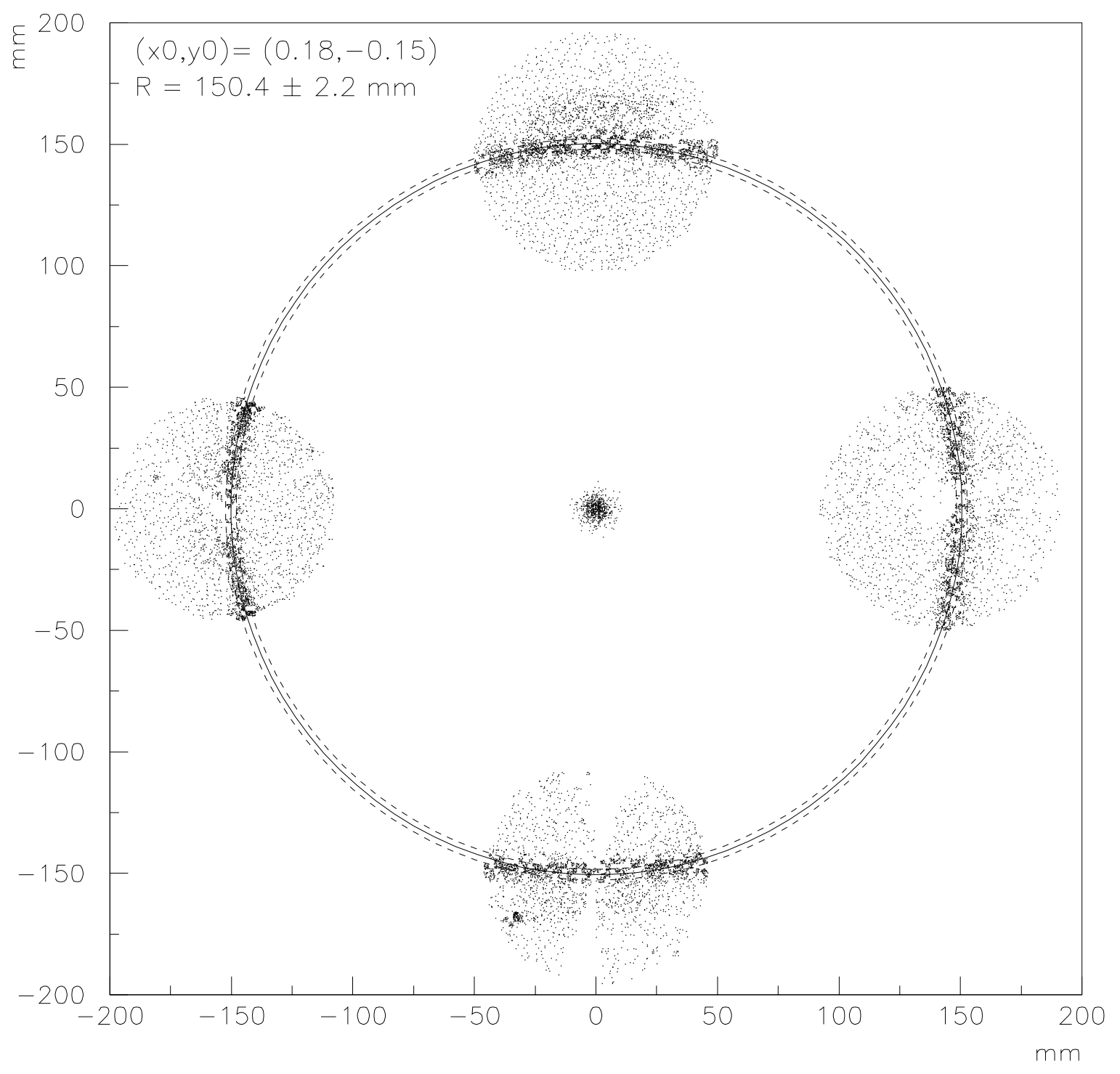

Figure 10: Display of the four HPDs response with the fitted Cherenkov ring for a negative pion particle beam of $9 \mathrm{GeV} / \mathrm{c}$ momentum. The data shown were taken with $4 \mathrm{~cm}$ aerogel and using the filter of the type D263. 

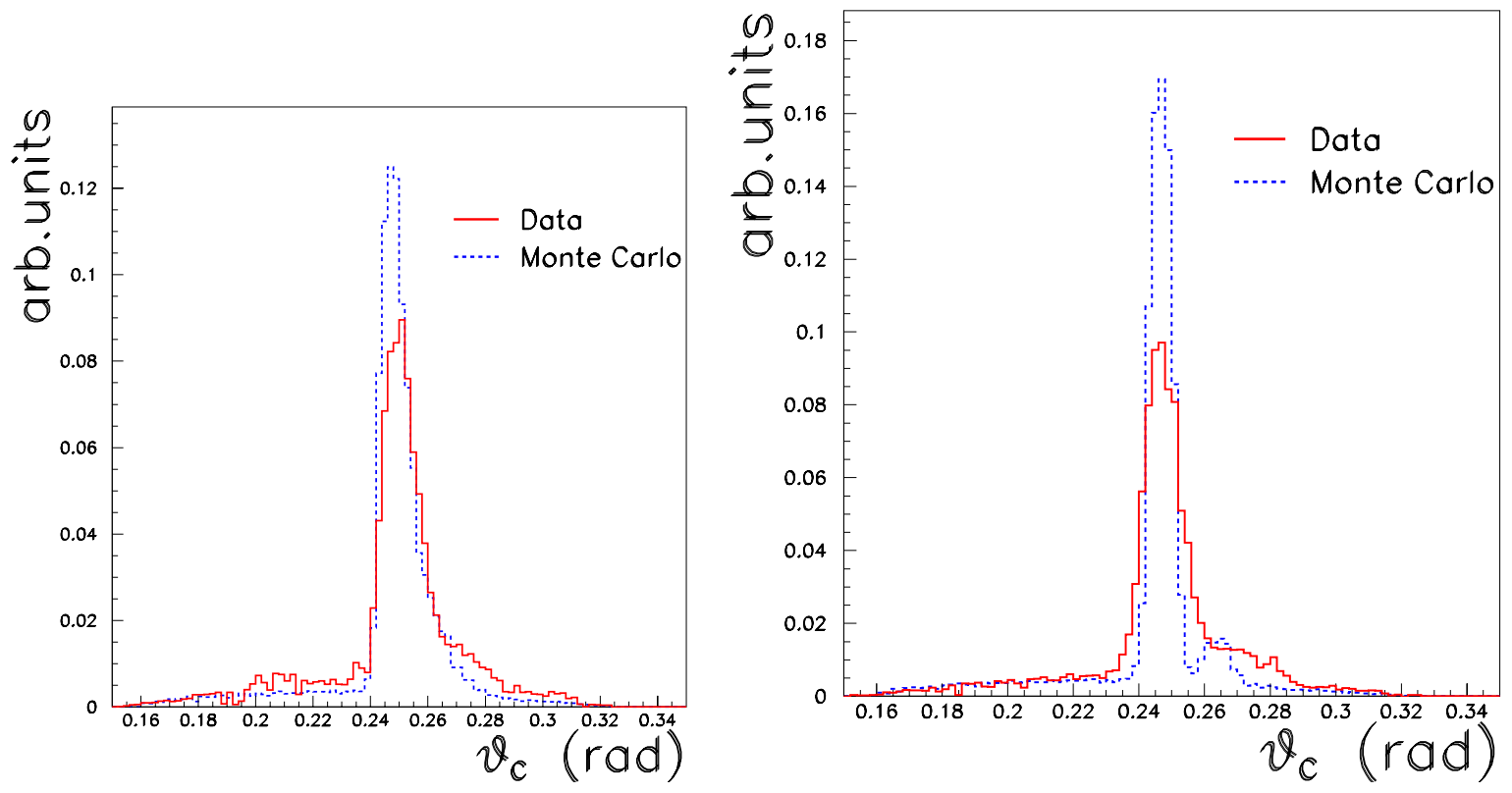

Figure 11: Distribution of the single photon Cherenkov angle for $4 \mathrm{~cm}$ Novosibirsk aerogel without (left) and with (right) filter D263. The dashed line corresponds to the MC expectation.
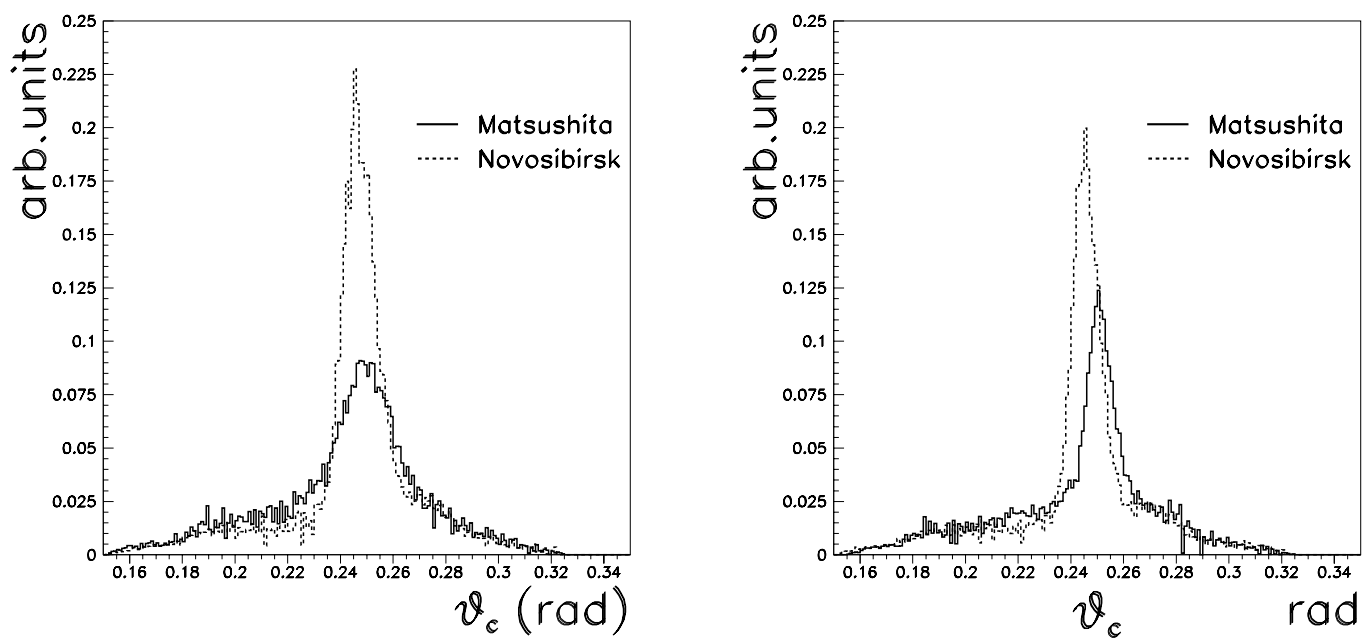

Figure 12: Cherenkov angle reconstructed in $8 \mathrm{~cm}$ thick samples of two different types of aerogel. Solid line is for Matsushita data, dotted line for Novosibirsk. Data taken without filter (left) and with D263 filter (right). 

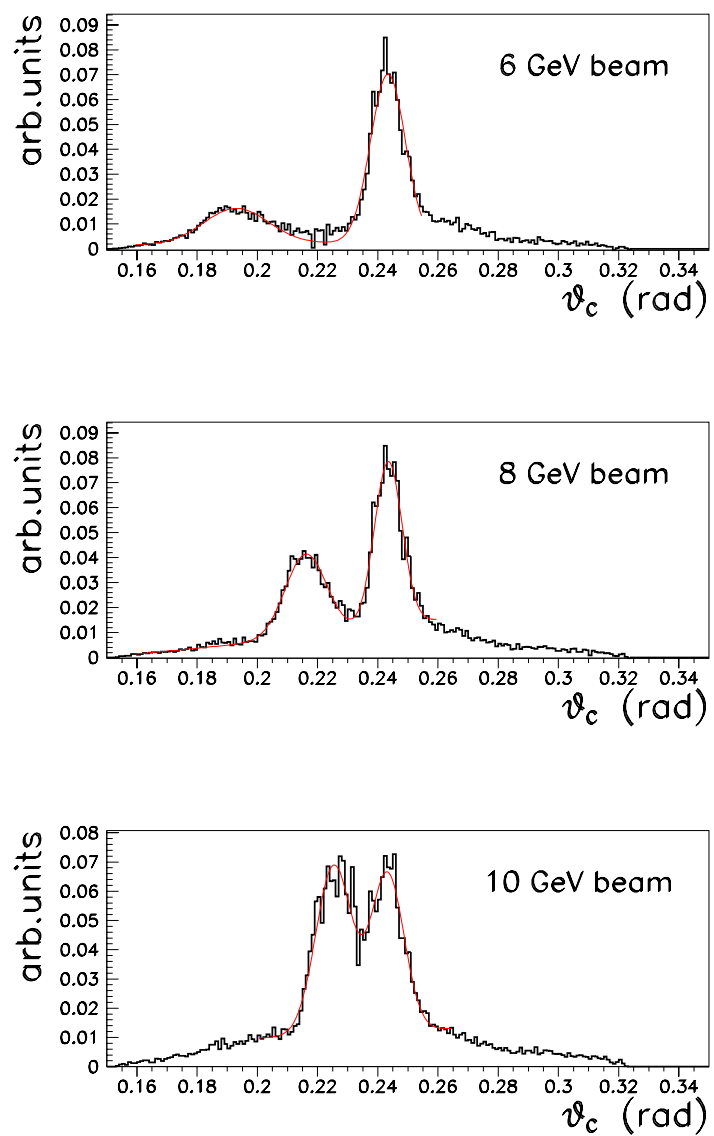

Figure 13: Single photon Cherenkov emission angle for $\pi^{+}$/proton beams of increasing energy from 6 to $10 \mathrm{GeV}$ and aerogel produced in Novosibirsk.

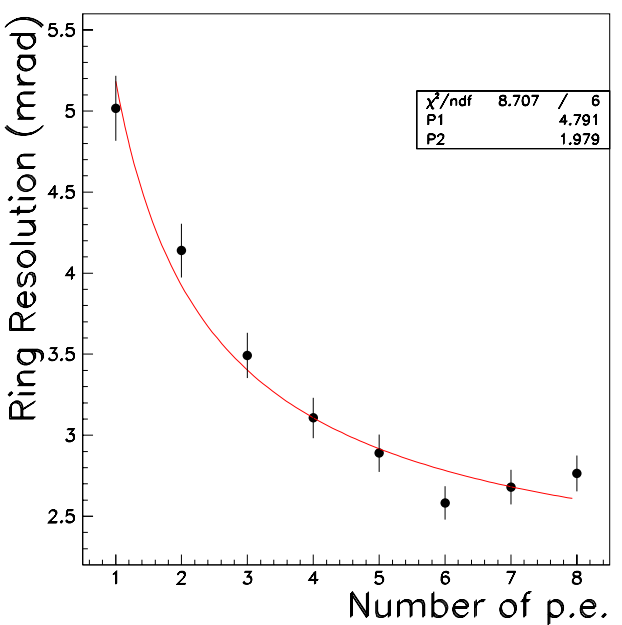

Figure 14: Resolution in mrad as a function of the number of detected photoelectrons. The curve shows the result of the fit of Eq. 3 to the data. 

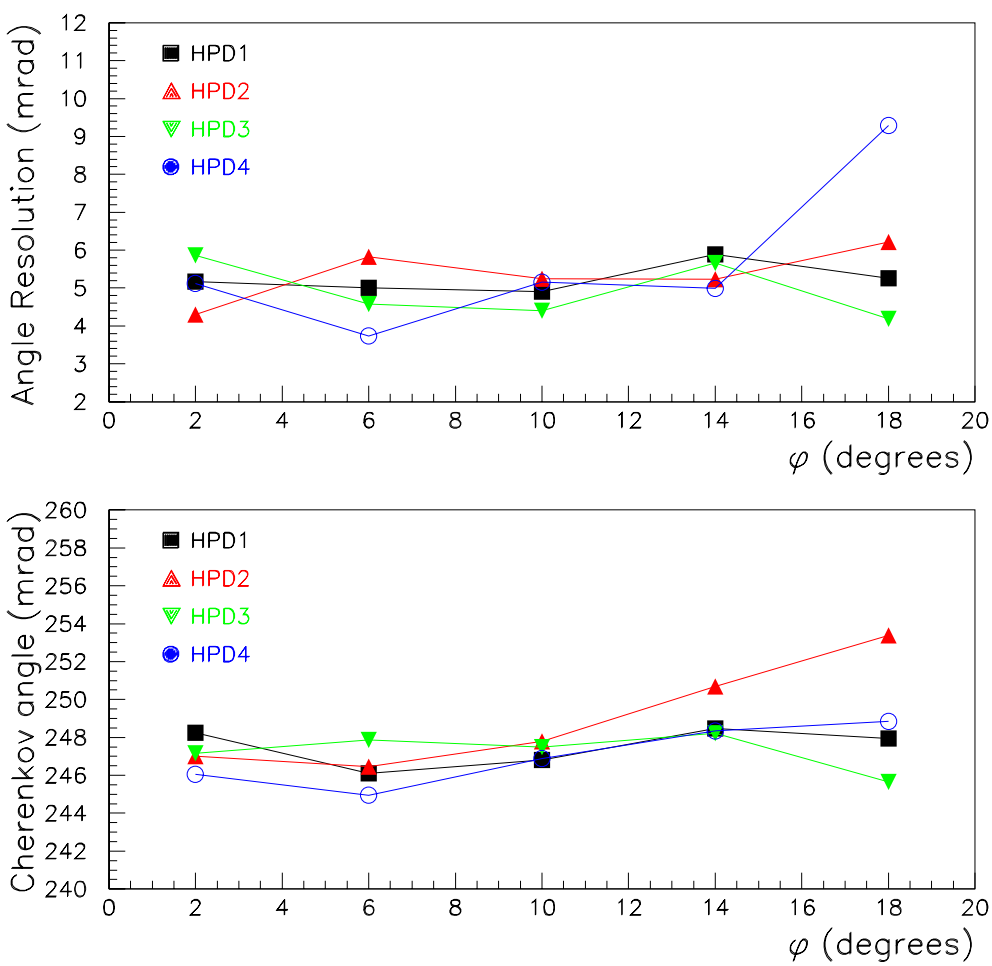

Figure 15: Top: resolution, in mrad, as a function of different azimuthal regions of the anode and for each photodetector, and (bottom) the reconstructed Cherenkov angle, in $\mathrm{mrad}$, in the same different regions. 